\title{
Rethinking organoid technology through bioengineering
}

\author{
Elena Garreta ${ }^{1,2,14}$, Roger D. Kamm ${ }^{\circledR 3,14}$, Susana M. Chuva de Sousa Lopes $\mathbb{1}^{4}$, Madeline A. Lancaster ${ }^{5}$, \\ Ron Weiss $\mathbb{D}^{6,7}$, Xavier Trepat ${ }^{8,9,10,11}$, Insoo Hyun ${ }^{12,13}$ and Nuria Montserrat $\mathbb{D}^{1,8,9 凶}$
}

In recent years considerable progress has been made in the development of faithful procedures for the differentiation of human pluripotent stem cells (hPSCs). An important step in this direction has also been the derivation of organoids. This technology relies on traditional three-dimensional culture techniques that exploit cell-autonomous self-organization responses of hPSCs with minimal control over the external inputs supplied to the system. The convergence of stem cell biology and bioengineering offers the possibility to provide these stimuli in a controlled fashion, resulting in the development of naturally inspired approaches to overcome major limitations of this nascent technology. Based on the current developments, we emphasize the achievements and ongoing challenges of bringing together hPSC organoid differentiation, bioengineering and ethics. This Review underlines the need for providing engineering solutions to gain control of self-organization and functionality of hPSC-derived organoids. We expect that this knowledge will guide the community to generate higher-grade hPSC-derived organoids for further applications in developmental biology, drug screening, disease modelling and personalized medicine.

"O ver the past two decades, stem cell research has advanced our understanding of key aspects of organogenesis through the exploitation of the self-organizing properties of adult stem cells (ASCs) and pluripotent stem cells (PSCs). Such progresses have led to the development of cell culture procedures to generate micro- and miniorgan-like structures on demand, the so-called organoids. In parallel, the emergence of the bioengineering field is leading to technological advances that allow proper instructive environments (physical and chemical), boosting cellular responses towards the formation of organ-specific multicellular structures in these miniorgan-like structures. Current methods rely on traditional three-dimensional (3D) culture techniques that exploit cell-autonomous self-organization of human PSCs (hPSCs) (Fig. 1). Nevertheless, hPSC-derived organoids still exhibit several short2 comings. These include the lack of reproducibility; lack of specificity with regard to cell-type(s) composition; uncontrolled size; shape heterogeneity; absence of proper vascular, immune and innervation components and organ-specific morphological features; and lack of functionality. Therefore, major goals of organoid technology are now focused on improving organoids cellular and morphological complexity (for example, via the induction of properly organized regional identities in brain organoids, or providing a developing branching collecting-duct system in kidney organoids), providing perfusable vascular networks (to facilitate organoid differentiation and lifespan, but also organoid-to-organoid connection for studying complex interactions between different tissue types), and enhancing organoid maturation in order to achieve relevant tissue-specific functionalities. Understanding and integrating self-formation capacities and programmability of hPSCs with bioengineering design may increase control of self-organization and functionality of hPSC-derived organoids. This knowledge will help the community to generate higher-grade organoids (in terms of cellular composition, architecture, function and reproducibility) for developmental biology, drug screening, disease modelling and personalized/precise medicine applications, and in the future to derive clinically relevant tissue-like structures for regenerative medicine applications (Fig. 1).

In this Review, we first look back on the historical origin of organoid technology and how early developments in 3D cell culture systems exploiting the self-organization ability of hPSCs have enabled generation of these powerful platforms. We will collectively examine how to apply current knowledge in organoid mechanics and transcriptomics to further control the arrangement function and composition of hPSC-derived organoids. Then, we foresee the immediate impact of engineering approaches (that is, biomimetic hydrogels, 3D bioprinting and microtechnologies) to overcome current hPSC-derived organoids challenges in the upcoming years. Finally, we will discuss on how ethicists, engineers and stem cell biologists will need to collaborate on engineering ethics and how this joint effort will benefit the success of the entire hPSC-derived organoid field.

\section{Engineering hPSC-derived organoids}

Insert some text here.

Evolution of 3D cultures in the organoid's history. Since the 1900s, developmental biologists have selected different model organisms to study the morphogenetic processes occurring during tissue and organ development, including sponges, amphibians,

'Pluripotency for Organ Regeneration, Institute for Bioengineering of Catalonia, the Barcelona Institute of Technology, Barcelona, Spain. ${ }^{2}$ University of Barcelona, Barcelona, Spain. ${ }^{3}$ Department of Biological Engineering and Department of Mechanical Engineering, Massachusetts Institute of Technology, Cambridge, MA, USA. ${ }^{4}$ Department of Anatomy and Embryology, Leiden University Medical Center, Leiden, the Netherlands. ${ }^{5} \mathrm{MRC}$ Laboratory of Molecular Biology, Cambridge Biomedical Campus, Cambridge, UK. 'Department of Biological Engineering, Massachusetts Institute of Technology, Cambridge, MA, USA. ${ }^{7}$ Synthetic Biology Center, Massachusetts Institute of Technology, Cambridge, MA, USA. ${ }^{8}$ Centro de Investigación Biomédica en Red en Bioingeniería, Biomateriales y Nanomedicina, Barcelona, Spain. ${ }^{9}$ Institució Catalana de Recerca i Estudis Avançats, Barcelona, Spain. ${ }^{10}$ Institute for Bioengineering of Catalonia, the Barcelona Institute of Technology, Barcelona, Spain. "Unitat de Biofísica i Bioenginyeria, Universitat de Barcelona, Barcelona, Spain. ${ }^{12}$ Department of Bioethics, School of Medicine, Case Western Reserve University, Cleveland, OH, USA. ${ }^{13} \mathrm{Center}$ for Bioethics, Harvard Medical School, Boston, MA, USA. ${ }^{14}$ These authors contributed equally: Elena Garreta, Roger D. Kamm. $\varpi_{e}$-mail: nmontserrat@ibecbarcelona.eu 

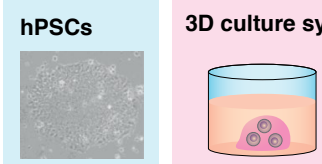

Cell embedding in matrigel

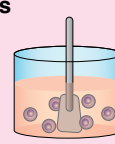

3D suspensio culture
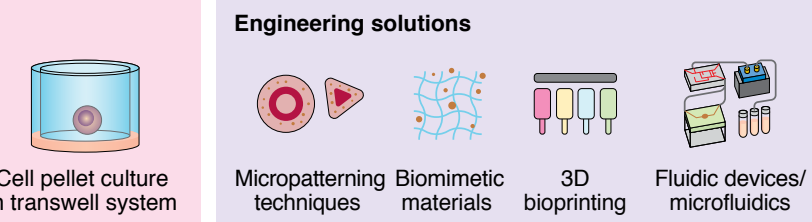

Emerging technologies

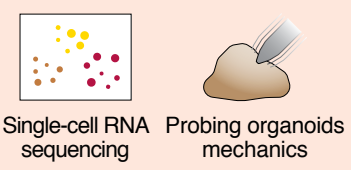

\begin{tabular}{|c|c|c|}
\hline 2006 & 2008 & 2010s \\
\hline L & & \\
\hline $\begin{array}{l}\text { Establishment } \\
\text { of hESCs and } \\
\text { hiPSCs }\end{array}$ & $\begin{array}{l}\text { 3D cortical } \\
\text { structures } \\
\text { (Eiraku, 2008) }\end{array}$ & $\begin{array}{c}\text { Differentiation methodologies } \\
\text { to generate hPSC-derived } \\
\text { organoids }\end{array}$ \\
\hline
\end{tabular}

structures
(Eiraku, 2008
2D micropatterns to guide hPSC self-organization

\section{Generation of self-organized cardiac micro- tissues (Ma, 2015)}

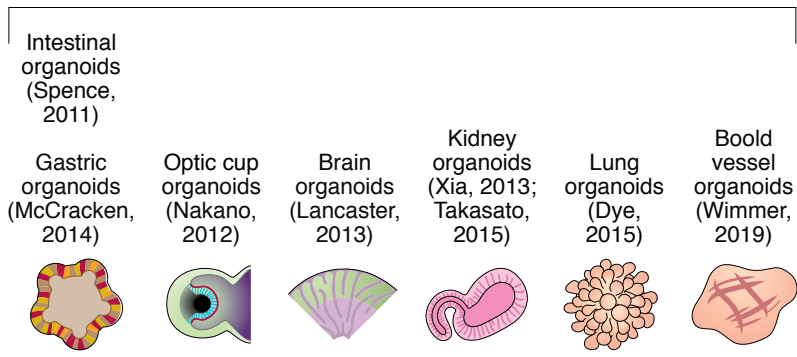

Generation Combination with a self-organizing microfluidic system to provide germ layers, so controlled morphogen called gastruloids gradients for hPSC (Martyn, 2018) differentiation (Manfrin, 2019)

\section{Brain organoid} formation in micro-structured scaffolds (Lancaster, 2017)
Compliant hydrogels

Use of synthetic PEG-based hydrogels accelerate the for intestinal $\quad$ formation of kidney organoids derivation organoids (Garreta, to control 3D organoid differentiation

Cruz-Acuña, 2017) 2019)

A physiological model Multi-layer human of the human motor retina-ona-chip platform unit for disease $\quad$ for drug screening modelling (Osaki, 2018) (Loskill, 2019)

mimic tissue-tissue interactions

$\begin{array}{lll}\text { Unveiling organoid } & \text { Mechanics of } & \text { An on-chip approach } \\ \text { mechanics for } & \text { self-organizing } & \text { for model ling the } \\ \text { disease mode } & \text { optic cups } & \text { physics of brain folding } \\ \text { (Okuda, 2018) } & \text { (Karzbrun, 2018) }\end{array}$

\section{Current organoid challenges:}

- Increase organoid lifespan

- Provide proper vascularization

- Achieve relevant tissufic architecture and functionality

\section{Bioengineering ethics}

- Informed consent requirements for procuring human cells

- Development of ethical guidelines for organoid research

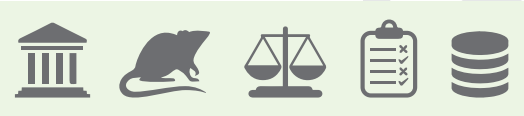

Novel ethical issues include:

- Biobanking and research sharing of organoids

- Potential role of organoids in personalized medicine

- Impact of organoids on animal research and clinical trials

Fig. 1 | Advances in engineering hPSC-derived organoids. Timeline of milestones for the generation of organoids from hPSCs. To date, several engineering solutions controlling self-organization, differentiation and tissue boundary conditions have explored micropatterning and microfluidics to improve organoid outcomes. Other approaches aiming to gain control on cell-to-cell and cell-to-ECM are considered when fabricating new materials emulating biochemical or biophysical properties of native tissues. These biomimetic materials can be further exploited for 3D bioprinting creating better tissue architectures. The application of these engineering approaches together with emergent technologies from the fields of transcriptomics and mechanics are expected to provide a better control of hPSC organoid generation. At the same time, the ethical dimension in this field compromises policies for patient consent, biobanking or animal use. All these considerations call for responsibility in communicating results to the public and the need to discuss these topics between ethicists, engineers and stem cell biologists.

27 chick and mice. All these different systems have contributed substantially to address key long-standing questions in this field. Groundbreaking observations performed in these organisms during the first decades of the last century highlighted the intrinsic capacities of tissues to follow predetermined developmental and functional programmes and how these dictated the mutual and Q exclusive relations between cells during reaggregation (Box 1). By the 1940s, different researchers demonstrated that growing tissue removed from the avian limb rudiment would rearrange and pattern in vitro and in ovo ${ }^{1,2}$. In addition, disaggregated and reaggregated chick embryonic kidneys became proper kidney epithelial tubules surrounded by mesenchyme-derived stroma in culture ${ }^{3}$. These experiments pinpointed that cell reaggregation could result in self-reassembly of tissue-like structures, whereby cells organized autonomously into pre-patterned structures. Paul Weiss and A. Cecil Taylor showed that when chick embryonic cells from different organs at advanced stages of development were reaggregated and grafted into a highly vascularized 'neutral environment', as the chick chorioallantoic membrane (CAM), the resulting 'cell masses' formed well-organized organs ex vivo ${ }^{4}$. Interestingly, in that study the authors highlighted the phenomenon of self-organization as the major cause driving morphogenesis instead of 'external inductions', which was the major trend explaining development at that time ${ }^{4}$.
By contrast, experiments by Clifford Grobstein demonstrated that when an inducing source, such as spinal cord, was reaggregated with the kidney metanephric mesenchyme, nephron-like structures were able to develop in vitro ${ }^{5}$.

As the knowledge of 3D cultures progressed, intense research was devoted to explore how the extracellular matrix (ECM) dictates morphogenesis and function in a wide range of cell cultures ${ }^{6}$. Later, Hans Clevers and colleagues applied this knowledge to culture single-cell suspensions of Lgr5+ mouse intestinal stem cells embedded in Matrigel under specific culture conditions succeeding in the generation of intestinal organoids with a crypt-villus architecture $^{7}$. This seminal study and others facilitated the development of ASC-derived organoids, such as stomach, pancreas, colon, prostate and liver. Progress in ASC-derived organoids and major advances in this field have recently been addressed in several excellent reviews ${ }^{8,9}$.

Since the isolation of human embryonic stem cells (ESCs) in $1998^{10}$ and the reprogramming of human somatic cells into induced pluripotent stem cells (iPSCs) ${ }^{11}$, both sources have become instrumental in recapitulating the fundamental principles of tissue differentiation and morphogenesis. Because PSCs represent the starting point of differentiation (pluripotency stage), they offer a model of organ ontogeny and a 'minimal system' for discerning in a systematic manner the relative contribution of different cellular components to 


\section{Box 1 | Evolution of 3D cultures in the organoid's history}

Q Reviewing the origin of organoids brings to mind an assay written by Henry Van Peters Wilson in 1907 entitled 'On some phe-

Q10 nomena of coalescence and regeneration in sponge ${ }^{100}$. Current key issues in the organoid field were already highlighted in his words: "In a recent communication I described some degenerative and regenerative phenomena in sponges and pointed out that a knowledge of these powers made it possible for us to grow sponges in a new way. The gist of the matter is that silicious sponges when kept in confinement under proper conditions degenerate in such a manner that while the bulk of the sponge dies, the cells in certain regions become aggregated to form lumps of undifferentiated tissue". H. V. Wilson refers to current concepts such as growing cells and tissues as "a new way" and stresses the need to define "proper conditions" to accomplish this goal. Later, in 1906, Ross G. Harrison pioneered the hanging drop culture system to study the origin of nerve cells by culturing fragments of frog embryonic nerves in a drop of lymph on a coverslip ${ }^{101}$. This approach allowed the direct observation of a growing nerve "while alive", setting the basis for other experiments culturing tissues of different embryonic origins for prolonged periods of time. By the 1950s, collective efforts led to the development of methods sustaining the culture of tissue fragments (through the watch glass method by Strangeways and Fell, 1926) ${ }^{102}$ and organ slides (through the lens paper method by Trowell, 1954) ${ }^{103}$. By the 1980s, the community had acquired technical skills and knowledge of ECM biology to continue exploring 3D cultures, a key feature to enable organoid culture. Among the first examples of what today might be considered an organoid is the work of Streuli and Bissell that pioneered the development of 3D mammary gland cultures ${ }^{104}$. Slight modifications of some of these 3D culture methods are nowadays applied to organoids ${ }^{14,18}$.

complex morphogenetic processes. Profiting from extensive knowledge accumulated from mouse development and mouse PSCs, research studies employ (extrapolated) growth signalling molecules to instruct hPSC differentiation in two-dimensional (2D) conditions or generating hPSC aggregate-like structures named embryoid bodies (EBs). This knowledge was key for obtaining for the first time self-patterned stratified cortical tissue after plating EBs generated in serum-free medium on a coated surface ${ }^{12}$. Later Sasai and colleagues maintained EBs as floating neuroepithelial cysts with minimal exogenously provided signals (Matrigel) ${ }^{13}$, which further self-organized into optic cup organoids containing spatially separated domains of neural retina and retinal pigmented epithelium. Building on this, further studies using spontaneous differentiation generated brain organoids with a wide variety of regional identities $^{14,15}$, while more directed approaches with small molecules could generate specific brain regions ${ }^{16,17}$. A common feature of the various methods developed for hPSC-derived neural organoids is that the factors that are applied to the cells attempt to reproduce the in vivo signalling networks and associated timing to which the rudimentary organ is exposed during development. Similarly, other hPSC-derived organoids seek to mimic these developmental events to generate tissues that mimic the in vivo counterparts. This has now been quite successful for kidney ${ }^{18}$, intestine ${ }^{19}$, lung $^{20}$ and inner ear ${ }^{21}$, to name a few.

Understanding self-organization and symmetry breaking. For an aggregate of cells (PSCs or committed organ progenitors) to evolve from a simple spheroid to an organoid with complex tissue architecture, symmetry breaking must occur. While the precise mechanisms that underlie this process are in most cases still unclear, in general it involves a certain degree of stochastic differentiation, cell sorting and feedback between neighbouring cells to set up boundaries and signalling centres ${ }^{22}$. This spontaneous organization probably results from stochastic fate acquisition, followed by cell rearrangements in which cells with similar adhesive properties tend to associate with one another ${ }^{23}$.

This type of symmetry breaking continues throughout development, both in vivo and in vitro. As cells of the same germ layer accumulate in segregated compartments often lined by basement membranes of ECM, they too generate (and respond to) signalling patterns, resulting in symmetry breaking to start forming specific substructures that will develop into the various organ precursors. Similarly, anterior-posterior symmetry breaking has also been shown in the so-called mouse gastruloids, which are essentially mouse EBs exposed to a short pulse of Wnt stimulation ${ }^{24,25}$. These aggregates do not epithelialize or cavitate like in vivo pre-gastrulating mouse embryos, but spontaneously develop a localized patch of Brachyury-positive cells, indicating a process like primitive streak formation. Importantly, the vast majority of gastruloids forms only one such patch, pointing to feedback between cells to develop and elongate along one axis, similar to in vivo ${ }^{26}$.

Once symmetry breaking has occurred, further signalling events from specialized regions called signalling centres help to further pattern and set up organ topography. For example, in the developing brain, dorsal and ventral signalling centres in the roof plate and floor plate signal through Wnt/Bmp and Shh pattern adjacent progenitors and influence their fate ${ }^{27}$. Interestingly, floor plate signalling centres have been shown to spontaneously form in neural tube organoids with a pulse of retinoic acid ${ }^{28}$. Likewise, the forebrain dorsal signalling centre, called the hem, has been shown to spontaneously form in brain organoids ${ }^{29}$. These signalling centres are necessary for proper tissue architecture and for the formation of boundary regions that separate functionally distinct units such as the choroid plexus, which generates the cerebrospinal fluid, and the cerebral cortex.

Finally, once organ or tissue progenitors are specified and take on a specific regional identity, their intrinsic architecture can further shape the tissue and force asymmetric structures. In the (small) intestine, the position of intestinal stem cells within the crypt means that more differentiated daughter cells are forced up into the villi, which of course is important for proper functioning of the (small) intestine ${ }^{30}$. Similarly, neural stem cells called radial glia in the developing brain have an elongated shape that extends from the internal brain ventricles to the outer surface. Because their cell bodies take up the internal ventricular zone entirely, more differentiated daughter cells are forced up, and in fact use the elongated basal processes of the radial glia to migrate out into the cortical plate ${ }^{31}$. This positioning is important for proper architecture of the grey matter and formation of the white matter, an organization that also dictates proper connectivity and functioning of the brain.

Bioengineering strategies to enhance complexity. The derivation of hPSC organoids mainly relies on the self-organization principle with minimal control over the external inputs supplied to the system. At the same time, the uncontrolled nature of these processes contributes to the high heterogeneity observed in the current organoid systems, which results in low reproducibility of hPSC differentiation among different laboratories ${ }^{32}$, the variability in the differentiation outcomes between distinct hPSC lines, and inter-experimental variation ${ }^{33}$. Engineering-controlled microenvironments by the presentation of both chemical (that is, growth factors and integrin-binding proteins) and physical instructions (applied forces, elastic modulus, geometry and Poisson's ratio of matrix) represent affordable approaches towards the programmability of these cues for a better control of organoid self-organization and differentiation. 
Engineering self-organization. During numerous developmental processes, gradients of relevant morphogens and physical cues continuously help to establish both the polarity and the diversity in the structures that emerge in vivo. In the same manner, in self-organizing organoid systems, successive local microenvironment modifications rapidly promote the emergence of complex cellular patterns, further driving organoid morphogenesis in vitro. Nevertheless, the control of these morphogenetic processes in self-organizing organoids can be solely achieved through externally imposing biochemical and biophysical boundaries to the system. Therefore, engineering approaches to control the initial cellular density, geometry/shape and size of cell aggregates, cell-ECM interactions and biochemical gradients would provide more accurate instructions for hPSC-derived organoids generation. Conversely, these strategies may also be exploited when aiming to alter the path of development to achieve a different outcome.

Micropatterning techniques have been used to control physical boundary conditions by the creation of confined 2D patterns with defined geometry and size in culture substrates. Using this $2 \mathrm{D}$ approach, hPSCs cultured in confined circular patterns in the presence of BMP4, generated a concentric pattern of cells from the different germ layers, the so-called human gastruloids ${ }^{34,35}$. Similarly, cardiac micro-tissues have been reproducibly induced by seeding hPSCs in circular patterns ${ }^{36}$. These studies revealed that the synergy between biochemical cues and biophysical cell confinement for directing cell fate responses tightly depends on pattern geometry and size (Fig. 2a).

In vivo, ECM dynamics can delineate tissue boundaries guiding tissue morphogenesis ${ }^{37}$. For example, ECM remodelling has been shown to play an essential role during branching morphogenesis, a key developmental process that dictates the functional architecture of organs such as the lung, kidney (ureteric bud), mammary gland and salivary gland (submandibular gland) ${ }^{37}$. Although ECM mechanical properties dictate cell behaviours such as proliferation and differentiation ${ }^{38}$, only a few studies have explored the use of synthetic materials emulating in vivo ECM for guiding hPSC-derived organoid formation ${ }^{39-41}$ (Fig. 2b,c). Of note, whereas most natural ECMs are nonlinear and viscoelastic ${ }^{42}$, many synthetic matrices are not. These characteristics and their effect on organoid self-organization remain under investigation.

A powerful approach for creating complex biomolecule gradients is the use of microfluidic technology. In a recent study a microfluidic device was used to generate orthogonal gradients of retinoic acid and smoothened agonist (a small molecule activator of the sonic hedgehog ( $\mathrm{SHH}$ ) pathway), either simultaneously or sequentially, that allowed the derivation of motor neurons from mouse ESC-derived EBs by emulating in vivo neural tube patterning $^{43}$. More recently, a microfluidic device was designed to expose hPSC colonies to spatiotemporally controlled morphogen gradients generated from artificial signalling centres that promoted the generation of different axially arranged differentiation domains in $2 \mathrm{D}$ hPSC cultures $^{44}$ (Fig. 2d). Soon, these approaches could be further advanced to control size and geometry of 3D cultures and their exposure to controlled morphogen gradients, which represents an additional layer of complexity yet to be overcome. In this regard, an elegant study implemented a $3 \mathrm{D}$ co-culture strategy for mimicking a developmental organizer, which consisted in the $3 \mathrm{D}$ co-culture of small clusters of hPSCs producing SHH protein gradients together with wild-type hPSCs. This resulted in the generation of polarized $\mathrm{SHH}$-patterned forebrain organoids that displayed distinct in vivo-like forebrain domains ${ }^{45}$ (Fig. 2e). Alternatively, the modulation of biochemical signalling in space and time could be achieved using dynamic hydrogel systems that change their chemi$\mathrm{cal}$ and mechanical properties over time due to light-triggered reactions. Soluble biomolecules would be locally tethered in a target area within the hydrogel network in a reversible manner, permitting the fabrication of very precise $3 \mathrm{D}$ patterns and gradients of biochemical cues that are dynamically controlled upon light exposure $^{46}$. These types of hydrogels could be useful to locally guide organoid self-organization (that is, by locally softening or stiffening of the matrix ${ }^{47}$, and even to create stiffness gradients ${ }^{48}$ ). However, further research is needed to fulfil the potential of this approaches in the organoid field.

Transcriptomics and tissue mechanics. The convergence of organoid technology with emergent transcriptomics and tissue mechanics techniques offer unprecedented opportunities to study human development and tissue morphogenesis as well as new prospects for personalized and precise medicine applications. To date most organoids (and organs) have been profiled or sequenced in bulk during the process of differentiation, but the field is moving rapidly towards a more in-depth knowledge of the cellular composition of organoids (and organs) ${ }^{49}$. In addition, the increased availability of organ-specific datasets together with the use of machine learning algorithms (that is, artificial intelligence) is making it possible to predict accurately the identity score of cell types present in organoids. One example of such algorithms is KeyGenes (http://www.keygenes.nl) ${ }^{50}$, which has been used to predict the proximity of kidney organoids to human fetal kidneys showing differentiation progression in time towards second trimester kidneys ${ }^{18,40}$. The dynamics of the molecular signalling pathways and tissue-specific transcription factors needed for each developmental transition in vivo is being revealed $^{51,52}$. In parallel, other technologies are also being developed to integrate positional information on top of genome-wide information such as in situ sequencing and high-multiplex imaging mass cytometry $^{53}$. These new technologies will be useful to associate positional information to cellular signatures and states within the organoid. When used at different time points during organoid formation these procedures have the potential to define a biochemical signature for each cell within the organoid. Furthermore, assessing differential responses in front of environmental or genetic manipulations during organoid ontogeny will also be important for capturing disease phenotype(s) (Fig. 3).

In vivo, a complex combination of genetic programmes and multiple types of microenvironmental cues instruct the shaping of tissues and organs during development. These interactions control a set of mechanical processes such as deformation, remodelling and flow, that ultimately shape the organ ${ }^{54}$. Our current understanding of the mechanics of morphogenesis has largely been obtained through top-down approaches, whereby the mechanisms that lead to shape changes can be deconstructed, for example, in whole embryos through a combination of imaging, genetic manipulations, and force inference methods $s^{54,55}$. While the relevance of these approaches is clear, using embryos has the drawback of limited accessibility in terms of imaging and mechano-chemical manipulation. In contrast, hPSC-derived organoids represent an affordable in vitro model for assessing both the mechanical aspects of human tissue morphogenesis and the impact of these processes during disease. In parallel with the development of tools to probe organoid mechanics (Box 2), the advance of computational tools and models that support mechanical measurements to predict and direct morphogenesis is crucial. To date, different model systems have been developed to explain the dynamics of flat epithelial monolayers, including lattice, phase fields, active network, particle and continuum models. Their application to three dimensions has already resulted in some successes ${ }^{56,57}$. These simulations can be used to study how cell-generated forces can give rise to folded epithelial shapes within organoids ${ }^{58}$. Indeed, very recently Karzbrun and colleagues have explored a similar approach to model the physics of the folding in the appearance of wrinkles during the in vitro development and self-organization of human brain organoids $^{59}$ (Fig. 3). 

2D cell confinement Micropatterning
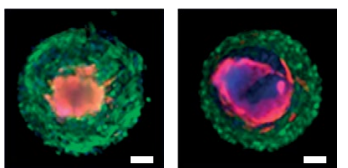

2D substrate stiffness Synthetic hydrogels

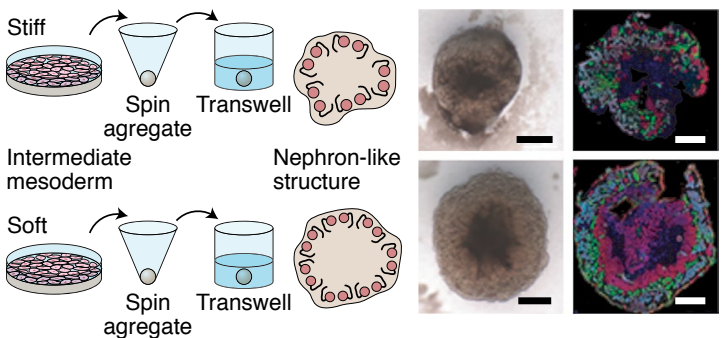

3D cell-ECM interactions

Micro-structured scaffolds
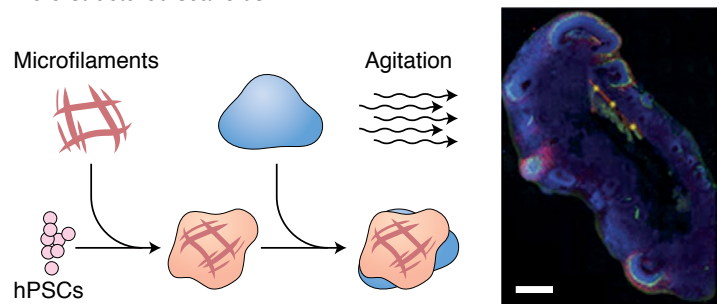

d

2D morphogen gradients Microfluidic devices

$\square$ Morphogen $\square$ Anterior progenitors $\square$ hPSCs $\quad \square$ Posterior progenitors
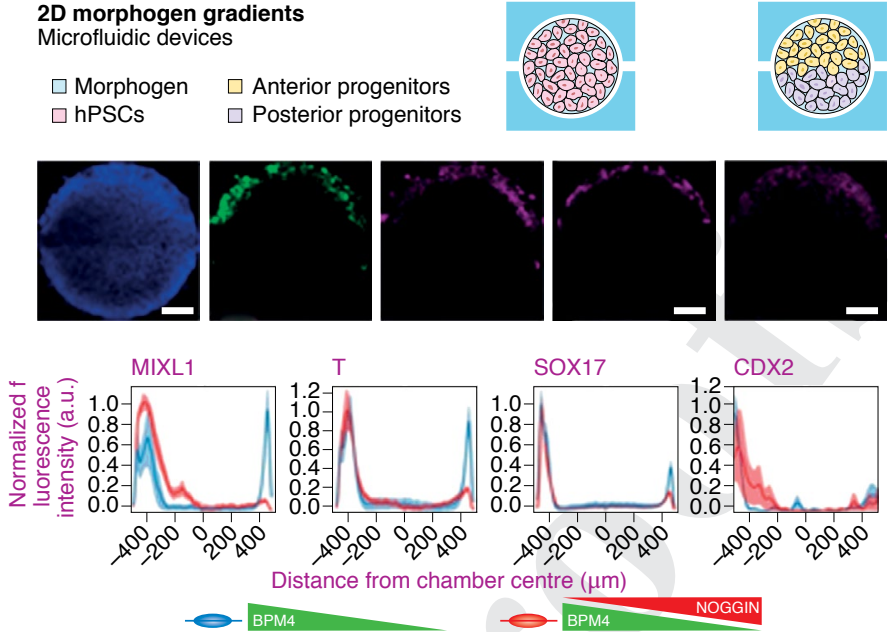

3D morphogen gradients

3D co-culture
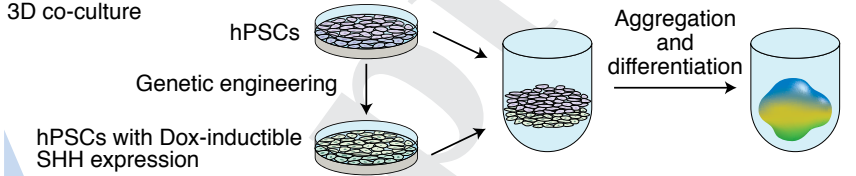

SHH expression
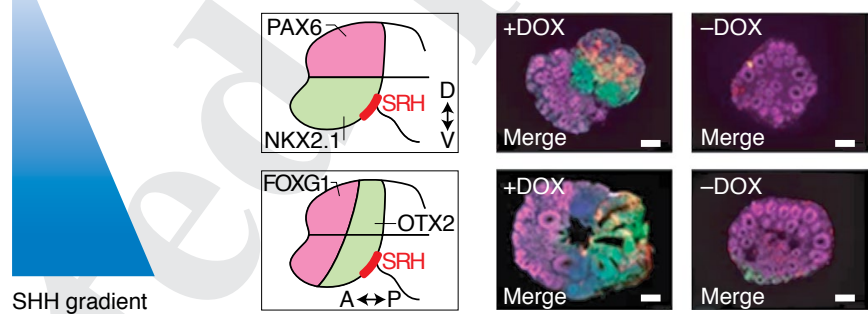

Fig. 2 | Engineering approaches to guide hPSC-derived organoids self-organization. a, Geometry and size of 2D patterns generated on substrates by micropatterning techniques have proved to influence hPSC self-organization and commitment into small cardiac like-structures through the generation of diffusible biochemical gradients and local mechanical cues. b. Strategies promoting cell-cell and cell-ECM interactions can also be applied to better direct hPSC-organoids differentiation. For example, the use of polyacrylamide hydrogels mimicking a soft microenvironment mechanically instructed the derivation of hPSC-renal progenitors (intermediate mesoderm) in a 2D setting, which after aggregation, showed higher potential to give rise to kidney organoids compared with rigid conditions. c, The use of micro-structured poly(lactic-co-glycolic) acid (PLGA) scaffolds induced the formation of brain organoids with an elongated shape that regionalized into different forebrain regions and further showed the development of aligned neurons, forming a pseudo-cortical plate structure. d, Other strategies involve incorporating mechanical and topographical cues in combination with biochemical signalling. The use of microfluidics for culturing size-controlled hPSC colonies generated precise morphogen gradients that instruct hPSC differentiation into different axially organized germ layer domains. e, 3D morphogen gradients were successfully generated through the co-culture of small clusters of genetically modified hPSCs that express SHH in an inducible manner, which upon aggregation together with wild type hPSCs, formed cell spheroids that polarized and further resulted into brain organoids with in vivo-like dorsal-ventral and anterior-posterior forebrain regions. In the next years strategies supporting the external control of biophysical and biochemical cues to guide hPSCs self-organization are expected to contribute to standardize hPSC organoid derivation. Figure reproduced with permission from: ref. ${ }^{36}$, Springer Nature Ltd (a); ref. ${ }^{40}$, Springer Nature Ltd (b); ref. ${ }^{41}$, Springer Nature Ltd (c); ref. ${ }^{44}$, Springer Nature Ltd (d); ref. ${ }^{45}$, Springer Nature Ltd (e). Illustrations adapted with permission from: ref. ${ }^{98}$, Springer Nature Ltd (b); ref. ${ }^{41}$, Springer Nature Ltd (c); ref. ${ }^{99}$, Springer Nature Ltd (e).

Overall, coupling transcriptional information and mechanical signatures of cells within the organoid in time and space will provide a precise description of the organoid dynamics and the emergence of tissue functionality during organoid ontogeny as well as in response to different environmental stimuli or genetic manipulations for studying human development and disease.

Bioengineering strategies to enhance maturation and function. Current organoid methodologies have allowed the formation of relatively complex tissue-like structures of limited lifespan and functionality. Importantly, the possibility to couple these approaches using patient specific iPSCs ${ }^{60,61}$ or through CRISPR/Cas9-mediated gene editing in founder hPSCs $s^{59,62}$ or in the time course of organoid differentiation ${ }^{63,64}$ has started to show the utility of these systems for disease modelling applications. The final realization of these systems imposes the development of engineering approaches to provide the necessary nutritional and gas exchange needs to allow increase in size, lifespan, complexity and maturation. While these strategies may offer the possibility to generate larger scale tissues from organoids, it may also allow different tissue types to connect for example via the embedding of vasculature.

Engineering vascularization. In most organoid culture systems, nutrient and gas exchange is achieved by passive diffusion via the culture media, thus limiting the cell aggregate size to $\sim 1 \mathrm{~mm}$ to prevent the formation of a necrotic core. In most higher-level organisms, this 


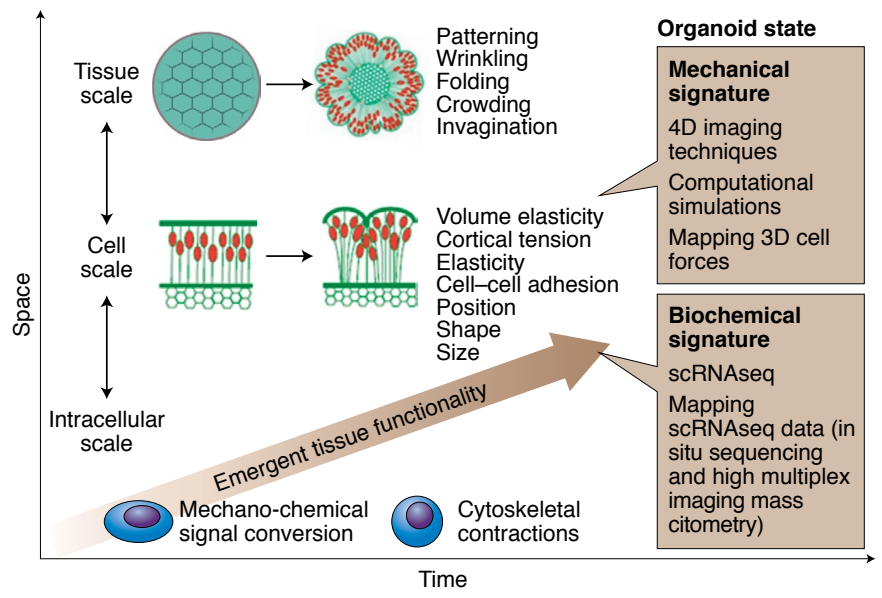

Fig. 3 | Integrating emergent technologies for understanding tissue morphogenesis. Single-cell transcriptomics techniques allow for high-resolution analysis of organoids heterogeneity and similarity to in vivo counterparts. Complimentary technologies as in situ sequencing and high multiplex imaging mass cytometry are being developed to associate cell positional information to cellular signatures. In parallel, the field of cell and tissue mechanics is developing new tools for probing 3D organoid mechanics coupled to robust computational models to further predict how cell-generated forces can shape organoids. In the near future, recent advances in multiplex measurements of mechanical and biochemical cellular signatures across a range of spatial, temporal and functional scales or in response to different environmental or genetic perturbations would precisely define organoid dynamics during hPSC organoid differentiation would offer an unprecedented scenario for investigating human development and disease. Illustrations for cell and tissue scale measurements reproduced with permission from ref. ${ }^{59}$, Springer Nature Ltd.

size constraint is overcome by the development of a perfused vasculature that enables the convective transport of nutrients and gases throughout the organ. To solve the problem of insufficient nutrient and gas supply, some studies have cultured organoids in suspension under continuous agitation or in spinning bioreactors ${ }^{65,66}$. One important issue when envisioning strategies for organoid vascularization is how to generate organ-specific endothelial cells that can properly organize and assemble to form organ-specific vasculature supporting organoid function. Remarkably, human blood vessel organoids have been recently derived from hPSCs ${ }^{67}$, but the role of specific inductive cues for later specification and differentiation into organ-specific endothelial cells needs further research. The gene regulatory networks that control endothelial cell specification in mouse brain, liver, lung and kidney has recently been described ${ }^{68}$. This information will be crucial when defining new approaches for the generation of organ-specific endothelial cells to be supplemented or endogenously generated in developing organoids. As a starting point, researchers have approached hPSC-organoid vascularization through the introduction of endothelial cells ${ }^{69}$ or using proangiogenic factors during the differentiation process ${ }^{70}$. Nevertheless, only a few studies have added support cells (that is, pericytes, smooth muscle cells or a stromal component ${ }^{71}$ ).

In vivo vascularization (host-derived vascularization) of either hPSC-derived cerebral organoids ${ }^{72}$, liver buds ${ }^{73,74}$ or kidney organoids ${ }^{75}$ has been partially achieved after organoid transplantation into immunodeficient mice. In a different approach, hPSC-derived kidney organoids have been engrafted onto the chick CAM showing host vascularization, but also the formation of capillary loop stage glomeruli-like structures with endothelial cells of human origin in close contact to podocyte-like cells ${ }^{40}$ (Fig. 4a). Altogether, these findings indicate that the formation of functional vascular

\section{Box 2 | Techniques for probing organoids mechanical properties}

There are several techniques to probe cell and tissue mechanical forces, but their applicability is mostly restricted to single cells and monolayers in vivo and in vitro ${ }^{105}$. Recently, some of them are being applied to organoids, but technical challenges remain substantial.

Genetically ${ }_{\Delta}$ encoded Förster resonance energy transfer sensors. This promising approach measures tension at specific molecules within adhesion sites or in the cytoskeleton ${ }^{106}$. These sensors are easy to (over)express and they provide molecular scale information. They do not provide directional information and their relatively low signal-to-noise ratio restricts applicability in 3D multicellular systems.

$3 \mathrm{D}$ traction force microscopy. It maps traction stresses at the interface between a cell and its surrounding ECM in 3D, computing the cell-induced deformation of the $\mathrm{ECM}^{107}$. Major limitations include the complexity of the used algorithms and the fact that the ECM properties must be known.

Insertion of cell-size soft droplets into the tissue. Because the mechanical properties of these inserted droplets are known, they can provide reliable measurements of physiological stress ${ }^{108}$. Its application is limited by the difficulty of micro-injecting the droplets into the organoid.

Laser ablation. A high-power laser pulse is used to ablate cellular structures and force is then computed from the resulting recoil of the tissue surrounding the ablation area ${ }^{109}$. This approach can be readily translated to organoids. It is an invasive technique and, therefore, is not suitable to measure the time evolution of forces.

Optical force inference technologies. They generate high-resolution imaging of cells and use force balance arguments and assumptions of material properties to infer the forces that cause a given change in shape ${ }^{110}$. They have an enormous potential to unveil the mechanical state of an organoid as it acquires a certain shape.

networks upon transplantation possibly require a synergistic modulation of the microenvironment together with the formation of the proper interactions between the host vasculature and the endothelial-committed cells (pre-)existing in the engrafted hPSC-derived organoids. This last point will probably prove to be both necessary and highly challenging in endodermal and ectodermal hPSC-derived organoids, where the endogenous formation of alternative mesenchymal fates (that is, endothelial cells) during the onset of differentiation is scarce or totally missing.

The use of microfluidic systems to engineer vascularization represents an alternative to in vivo transplantation methodologies. In vitro microvasculature is being created by encapsulating endothelial cells within a hydrogel introduced into a microfluidic device that self-assemble into perfusable vascular networks. This approach has been further explored to create models for the blood-brain barrier ${ }^{76}$ (Fig. 4b), tumour angiogenesis ${ }^{77}$ and tumour cell extravasation ${ }^{78}$. Interestingly, in a recent study a $3 \mathrm{D}$-printed millimetre-size chamber was designed to culture hPSC-derived kidney organoids under constant flow. This approach sufficed for the expansion of endothelial progenitors within the organoids leading to a higher degree of vascularization compared to the static culture conditions ${ }^{79}$. Another possibility to create vascular networks is through the fabrication of perfusable channels by $3 \mathrm{D}$ bioprinting, as recently developed through the combination of EBs containing iPSC-derived cardiac myoblasts and cardiac fibroblasts with a sacrificial 3D-printed vascular network ${ }^{80}$. The fabrication of the final construct through this 'organ-building blocks' approach reached cell densities comparable 

In vivo transplantation approaches Liver bud Brain organoid

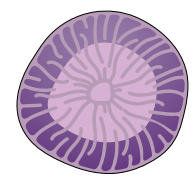

$\searrow$

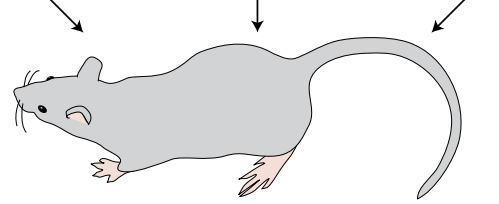

Vascularized liver bud
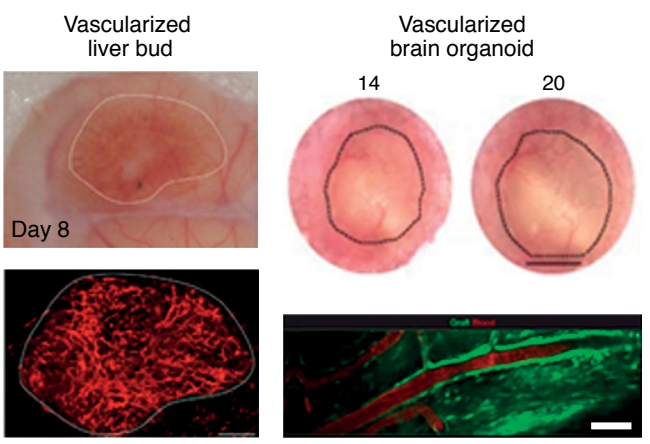

Vascularized
brain organoid

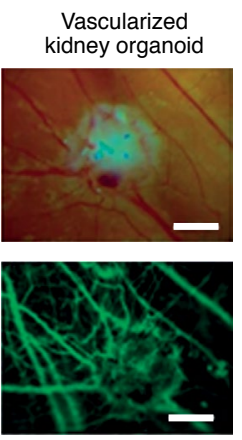

Kidney organoid
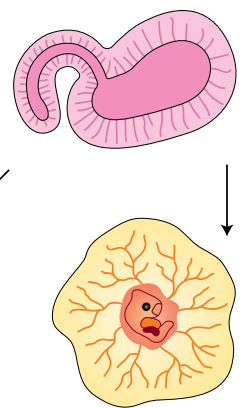

b

\section{Bioengineering approaches}

Blood-brain barrier on a chip

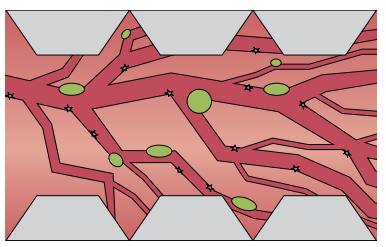

Kidney organoid on a chip

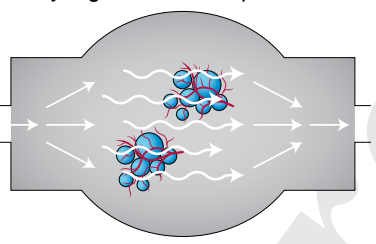

Vasculature by design

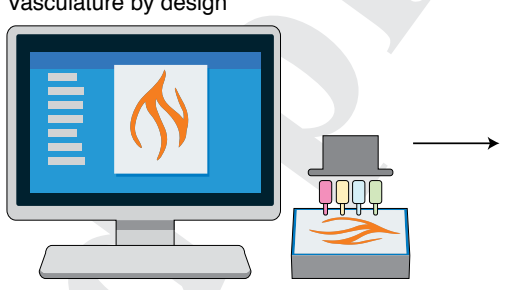

3D self-organized blood-brain barrier model

Fig. 4 | Strategies for engineering hPSC-derived organoids vascularization. a, Different in vivo transplantation approaches have been developed to incorporate vascular supply to hPSC organoids. Liver buds were transplanted into the cranial window of immunodeficient mice showing perfusion of human

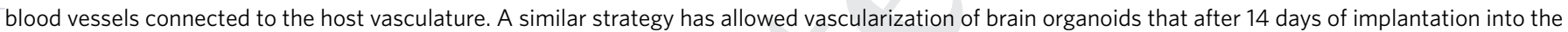
adult mouse brain cortex, leading to enhanced neuronal activity in the absence of necrosis. In a different approach, the chick chorioallantoic membrane was

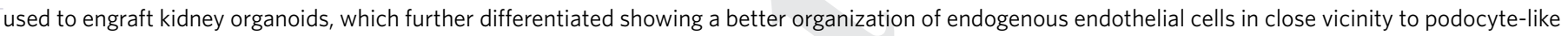
cells, similar to in vivo early-stage capillary loop formation in the maturing glomeruli. b, Bioengineering approaches have been explored for organoid

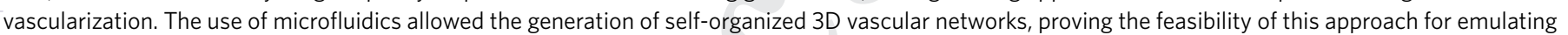
blood-brain barrier OoC from hPSCs. In another approach, a millimetre-size fluidic device was fabricated to culture kidney organoids under high fluid shear stress that substantially enhanced the formation of vascular structures composed of endothelial cells endogenously derived during kidney organoid

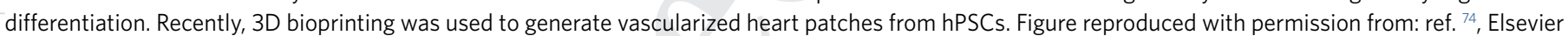
(a, vascularized liver bud images); ref. ${ }^{72}$, Springer Nature Ltd (a, vascularized brain organoid images); ref. ${ }^{40}$, Springer Nature Ltd (a, vascularized kidney

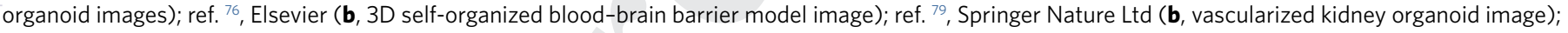
ref. ${ }^{81}$, Wiley (b, bioprinting tissue scale vasculature image).

to that found in living tissue ( 200 million cells per $\mathrm{ml})$ with macroscopic dimensions ${ }^{80}$. While the vascular diameters remained large compared to natural capillaries, the network could be patterned to replicate those of specific organs, as recently shown for heart ${ }^{81}$ (Fig. 4b). Another technical challenge, regardless of the approach used to induce vascularization, is to achieve stable and mature microvascular networks within organoids, meaning that the constructed vascular network should remain stable in time and have the proper composition and morphology of the native vasculature, even after removal of the stimuli used to induce vascularization. Overall, these bioengineering approaches could provide vasculature to organoids and offer control over nutrient/gas delivery and waste removal. This last point is of extremely importance when aiming to increase organoid lifespan, size and complexity compared to current static culture systems.

Engineering tissue-tissue interactions. During development, the shape of organs is regulated by biochemical and biophysical signals from adjacent developing tissues. These complex tissue-tissue interactions in vitro can be mimicked through spatial pre-patterning of organoids and through a variety of $3 \mathrm{D}$ co-culture approaches. The group of Ryuichi Nishinakamura has pioneered the generation of mouse PSC-derived ureteric bud progenitor cells and nephron progenitor cells, that were further manually assembled together with isolated mouse stromal cells generating kidney organoids mimicking the organotypic architecture of the embryonic kidney ${ }^{71}$. In the future, integrating native or PSC-derived branching epithelium into developing kidney organoids in the presence of stromal cells through 3D bioprinting may represent an affordable approach to precise positioning of these different components, so that the branching epithelium can act as an organizer of tissue geometry during kidney organoid differentiation. In a similar approach, using distinct committed cell progenitors as a starting point, Takebe and colleagues achieved the derivation of liver buds ${ }^{73,74}$. Other organoid systems that may benefit from the application of 3D bioprinting are those needing the interaction of cells coming from different embryonic lineages. A recent example is the generation of intestinal tissues with a functional enteric nervous system ${ }^{82}$ (Fig. 5). In another elegant study, Birey and colleagues have mimicked brain interregional interactions by in vitro 3D assembling pre-differentiated dorsal and ventral forebrain spheroids from hPSCs referred as 'brain assembloids', that proved to reproduce the saltatory migration of 


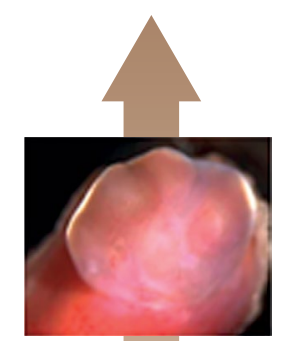

Innervated gut organoid

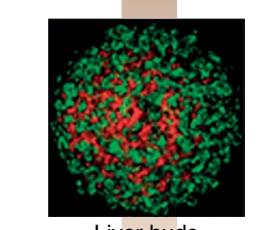

Liver buds

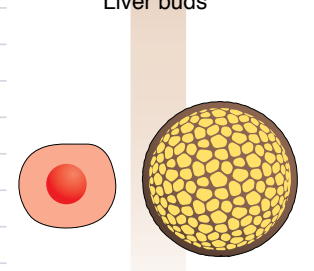

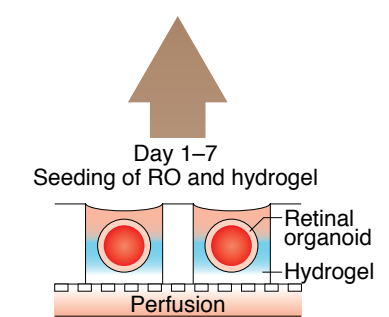

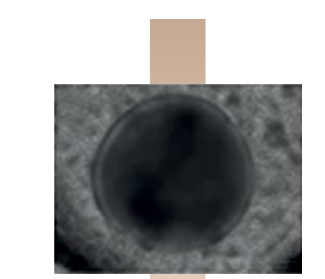

Retina on a chip

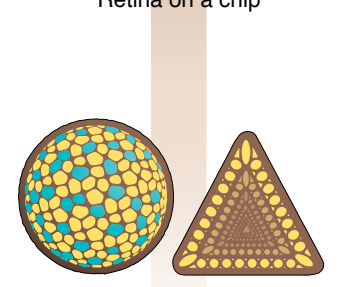

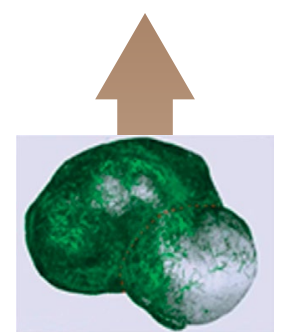

Brain assembloid

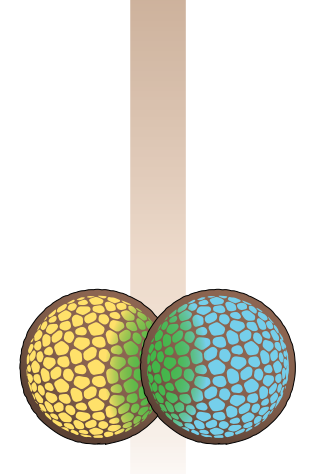

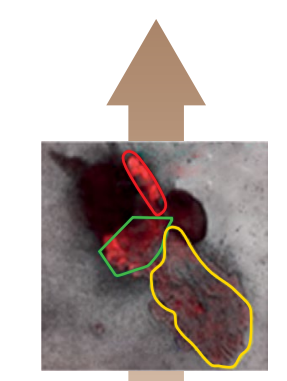

Hepato-biliary-
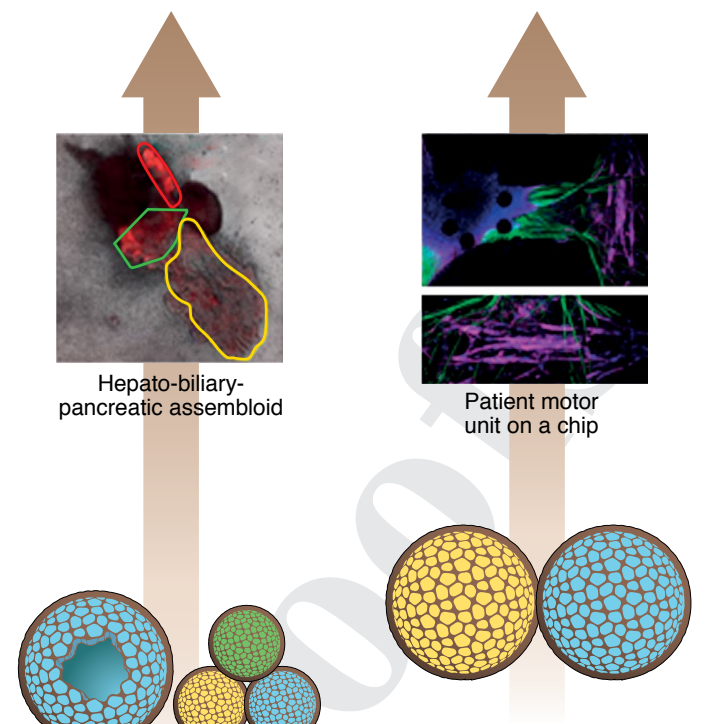
pancreatic assembloid

.

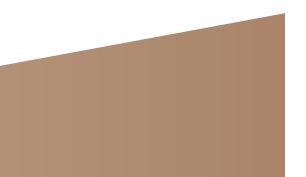

Organoid by design

Fig. 5 | Approaches for engineering tissue-tissue interactions. Forcing cell-to-cell contact by co-culturing in three dimensions in different combinations of cells and cell spheroids from hPSCs results in the self-assembly of complex 3D structures. These approaches can guide the formation of higher-grade organoids in terms of cellular, morphological and functional complexity, mimicking in vivo tissue-tissue interactions. The adaptation of microfluidic designs to organoid culture requirements has proved successful when fabricating OoCs suitable for drug screening and disease modelling applications. Figure reproduced with permission from: ref. ${ }^{82}$, Springer Nature Ltd (innervated gut organoid image); ref. ${ }^{73}$, Springer Nature Ltd (live buds image); ref. ${ }^{88}$ eLife Sciences (retina on a chip image); ref. ${ }^{83}$, Springer Nature Ltd (brain assembloid image); ref. ${ }^{84}$, Springer Nature Ltd (Hepato-biliary-pancreatic assembloid image); ref. ${ }^{87}$, AAAS (patient motor unit on a chip image).

interneurons observed in the fetal forebrain ${ }^{83}$. Importantly, this functional characteristic was abnormal when a neurodevelopmental disorder called Timothy syndrome was modelled using this assembloid system ${ }^{83}$. Following a similar approach, Takebe and colleagues recapitulated the human hepato-biliary-pancreatic tissue-tissue boundaries in vitro ${ }^{84}$ (Fig. 5).

Overall, these methodologies will require further optimization to achieve proper cells-to-organoid and organoid-to-organoid integration with the capability to recapitulate in vivo development and growth into higher complex and functional tissue structures.

Engineering physiological-like environments. Physico-chemical stimuli from systemic origin (that is, electrical stimuli, tissue strain and compression, fluid flow) are known to cooperatively participate to drive organ and tissue morphogenesis, maturation, and homeostasis. Microengineered devices mimicking physiological microenvironments have been exploited to create miniaturized cellular models, so called organ-on-a-chip (OoC), that can theoretically capture key aspects of (human) organ physiology. This technology holds the promise to recreate rather simple (2D), but dynamic physiological microenvironments. The recent adaptation of microfluidic devices to incorporate 3D cultures have accelerated their application for organoid research to harness tissue-tissue interactions and further organoid differentiation and maturation. Seminal studies have simulated the interaction of two-organ systems by using integrated tissue chambers, including gastrointestinal tract epithelium and liver cells to mimic liver injury ${ }^{85}$ or the interaction of human pancreatic islets and liver spheroids to recreate a type 2 diabetes model ${ }^{86}$. Another work designed a compartmentalized unit from hPSCs that consisted of a motor neuron spheroid activating contractile muscle in a model of amyotrophic lateral sclerosis (ALS) ${ }^{87}$. More recently, hPSC-derived retinal pigmented epithelial cells were assembled in close contact to hPSC-derived optic cup organoids in a multilayered $\mathrm{OoC}$ device proving the feasibility of this approach for drug toxicity screenings ${ }^{88}$ (Fig. 5). Moreover, by supporting physiological flow rates OoC have improved the growth and differentiation characteristics of hPSC-derived pancreatic islets ${ }^{89}$, intestinal ${ }^{90}$, stomach $^{91}$ and liver ${ }^{92}$ organoids.

Overall, these studies highlight the utility of the OoC technology to recreate tissue-tissue crosstalk and show the potential for advanced in vitro platforms for pharmacological testing. Also, coupling OoC systems may increase our understanding of complex inter-organ (that is, body-on-a-chip) behaviour in health versus disease. In the future, these connections might be accomplished via a self-assembled vascular network, thus enabling a more physiological context.

\section{Engineering ethics for hPSC-derived organoids}

Despite the recent advent of human organoid research, several pertinent ethical issues have already been identified by the research ethics and scientific literature. Among these are the informed consent requirements for ethically acceptable procurement of human cells necessary to generate organoids, the biobanking and transfer of patient-specific organoids for research, the impact of organoid technology on animal research and clinical trials, and the potential role of organoids in personalized medicine ${ }^{93,94}$. Much overlap 
exists between these and ethical issues surrounding other forms of biomedical research using cell lines and biospecimens. However, as engineers and stem cell biologists seek further opportunities to collaborate in organoid mechanics, there is a need to develop a new variant of traditional research ethics, that might be termed bioengineering ethics ${ }^{95}$.

Modelled after the ethics of design engineering pioneered by Dutch scholars, bioengineering ethics could emerge as a new discipline, characterized by encouraging ethicists and scientists to discuss the ethical choices and value trade-offs that drive research decisions during the design phase of experiments ${ }^{96}$. This approach recognizes that organoid bioengineering, like all other forms of engineering, is not a value-neutral activity. Bioengineering ethics calls for the integration of both bioengineering and ethics, so that ethicists and bioengineers can act as co-designers of research trajectories and choices made at the bench side, with each party informing the other, thus helping to infuse ethical reflection upstream during the development phases of new organoid projects.

Promoting this co-evolution of bioengineering ethics, however, will require high-level attention and dedicated resources from departments, universities and funding agencies ${ }^{97}$. Multi-level institutional mechanisms and incentives are needed to make such collaborations possible if ethicists and bioengineers are to be empowered to work together during the lifecycle of organoid research projects. For example, universities and funding agencies should allocate time and resources to enable ethicists, researchers and trainees to collaborate in the manner that bioengineering ethics demands, and to reward their efforts by establishing norms and financial structures that recognize their mutually supportive work. These challenges, though difficult, are tractable. Attention to these issues will foster a productive and responsible partnership between the disciplines of bioengineering and human organoid research.

\section{Future directions for hPSC-derived organoids engineering}

Despite the rapid progress in the field of organoid engineering the community is facing long term challenges as the absence of experimental and conceptual access to organoid mechanics. Coupled to the development of designer matrices with fine tunable and controlled biophysical properties all these developments will be essential to provide the proper physiological signals to drive stem cell self-organization and patterning without restricting later morphogenesis events ${ }^{38}$. On the other hand, the application of transcriptomics will reveal the complexity of cell types produced in organoids, the proportion of a specific cell population with respect to the others, as well as their differentiation state and spatial distribution. All this information will ultimately help to improve the efficacy, reproducibility and robustness of current hPSC-organoid differentiation procedures. Moreover, the definition of suitable scaling-up principles to maintain absolute values of physiological parameters and proper relative proportions between different types of cells within organoids may help to better mimic in vivo physiological conditions. Enhancing control over multiple tissue-tissue interactions when interrogating organ-like biological functions under homeostasis and disease-like conditions will ultimately support the application of hPSC-organoid technology for a new era in personalized and precise medicine applications.

Received: 20 December 2019; Accepted: 18 August 2020;

\section{References}

1. Rudnick, D. Regulation and localization in the hind limb bud of the chick embryo. Anat. Rec. 94, 492 (1946).

2. Saunders, J. W. An experimental study of the distribution, orientation, and tract specificity of feather germs in the wing of the chick embryo. Anat. Rec. 99, 647 (1947).
3. Moscona, A. \& Moscona, H. The dissociation and aggregation of cells from organ rudiments of the early chick embryo. J. Anat. 86, 287-301 (1952).

4. Weiss P, T. A. Reconstitution of complete organs from single-cell suspensions of chick embryos in advanced stages of differentiation. Proc. Natl Acad. Sci. USA 46, 1177-1185 (1960).

5. Auerbach, R. \& Grobstein, C. Inductive interaction of embryonic tissues after dissociation and reaggregation. Exp. Cell Res. 15, 384-397 (1958).

6. Simian, M. \& Bissell, M. J. Organoids: a historical perspective of thinking in three dimensions. J. Cell Biol. 216, 31-40 (2017).

7. Sato, T. et al. Single Lgr5 stem cells build crypt-villus structures in vitro without a mesenchymal niche. Nature 459, 262-265 (2009).

8. Clevers, H. Modeling development and disease with organoids. Cell 165, 1586-1597 (2016).

9. Xia, Y. \& Izpisua Belmonte, J. C. Design approaches for generating organ constructs. Cell Stem Cell 24, 877-894 (2019).

10. Thomson, Ja. et al. Embryonic stem cell lines derived from human blastocysts. Science 282, 1145-1147 (1998).

11. Takahashi, K. et al. Induction of pluripotent stem cells from adult human fibroblasts by defined factors. Cell 131, 861-872 (2007).

12. Eiraku, M. et al. Self-organized formation of polarized cortical tissues from ESCs and its active manipulation by extrinsic signals. Cell Stem Cell 3, 519-532 (2008).

13. Eiraku, M. et al. Self-organizing optic-cup morphogenesis in three-dimensional culture. Nature 472, 51-58 (2011).

14. Lancaster, M. A. et al. Cerebral organoids model human brain development and microcephaly. Nature 501, 373-379 (2013).

15. Quadrato, G. et al. Cell diversity and network dynamics in photosensitive human brain organoids. Nature 545, 48-53 (2017).

16. Pasca, A. M. et al. Functional cortical neurons and astrocytes from human pluripotent stem cells in 3D culture. Nat. Methods 12, 671-678 (2015).

17. Sakaguchi, H. et al. Generation of functional hippocampal neurons from self-organizing human embryonic stem cell-derived dorsomedial telencephalic tissue. Nat. Commun. 6, 8896 (2015).

18. Takasato, M. et al. Kidney organoids from human iPS cells contain multiple lineages and model human nephrogenesis. Nature 526, 564-568 (2015).

19. Spence, J. R. et al. Directed differentiation of human pluripotent stem cells into intestinal tissue in vitro. Nature 470, 105-110 (2011).

20. Dye, B. R. et al. In vitro generation of human pluripotent stem cell derived lung organoids. eLife 2015, e05098 (2015).

21. Koehler, K. R. et al. Generation of inner ear organoids containing functional hair cells from human pluripotent stem cells. Nat. Biotechnol. 35, 583-589 (2017).

22. Lancaster, M. A. \& Knoblich, J. A. Organogenesis in a dish: modeling development and disease using organoid technologies. Science 345 , 1247125 (2014).

23. Armstrong, P. B. Cell sorting out: the self-assembly of tissues in vitro. Crit. Rev. Biochem. Mol. Biol. 24, 119-149 (1989).

24. van Den Brink, S. C. et al. Symmetry breaking, germ layer specification and axial organisation in aggregates of mouse embryonic stem cells. Develeopment 141, 4231-4242 (2014).

25. Ten Berge, D. et al. Wnt signaling mediates self-organization and axis formation in embryoid bodies. Cell Stem Cell 3, 508-518 (2008).

26. van den Brink, S. C. et al. Single-cell and spatial transcriptomics reveal somitogenesis in gastruloids. Nature 582, 405-409 (2020).

27. Dessaud, E., McMahon, A. P. \& Briscoe, J. Pattern formation in the vertebrate neural tube: a sonic hedgehog morphogen-regulated transcriptional network. Development 135, 2489-2503 (2008).

28. Meinhardt, A. et al. 3D reconstitution of the patterned neural tube from embryonic stem cells. Stem Cell Reports 3, 987-999 (2014).

29. Renner, M. et al. Self-organized developmental patterning and differentiation in cerebral organoids. EMBO J. 36, 1316-1329 (2017).

30. Barker, N. et al. Identification of stem cells in small intestine and colon by marker gene Lgr5. Nature 449, 1003-1007 (2007).

31. Rakic, P. Extrinsic cytological determinants of basket and stellate cell dendritic pattern in the cerebellar molecular layer. J. Comp. Neurol. 146, 335-354 (1972).

32. Volpato, V. et al. Reproducibility of Molecular Phenotypes after Long-Term Differentiation to Human iPSC-derived neurons: a multi-site omics study. Stem Cell Reports 11, 897-911 (2018).

33. Phipson, B. et al. Evaluation of variability in human kidney organoids. Nat. Methods 16, 79-87 (2019).

34. Warmflash, A., Sorre, B., Etoc, F., Siggia, E. D. \& Brivanlou, A. H. A method to recapitulate early embryonic spatial patterning in human embryonic stem cells. Nat. Methods 11, 847-854 (2014).

35. Martyn, I., Kanno, T. Y., Ruzo, A., Siggia, E. D. \& Brivanlou, A. H. Self-organization of a human organizer by combined Wnt and nodal signaling. Nature 558, 132-135 (2018).

36. Ma, Z. et al. Self-organizing human cardiac microchambers mediated by geometric confinement. Nat. Commun. 6, 7413 (2015). 
37. Kim, H. Y. \& Nelson, C. M. Extracellular matrix and cytoskeletal dynamics during branching morphogenesis. Organogenesis 8, 56-64 (2012).

38. Vianello, S. \& Lutolf, M. P. Understanding the mechanobiology of early mammalian development through bioengineered models. Dev. Cell 48, 751-763 (2019).

39. Cruz-Acuña, R. et al. Synthetic hydrogels for human intestinal organoid generation and colonic wound repair. Nat. Cell Biol. 19, 1326-1335 (2017).

40. Garreta, E. et al. Fine tuning the extracellular environment accelerates the derivation of kidney organoids from human pluripotent stem cells. Nat. Mater. 18, 397-405 (2019).

41. Lancaster, M. A. et al. Guided self-organization and cortical plate formation in human brain organoids. Nat. Biotechnol. 35, 659-666 (2017).

42. Storm, C., Pastore, J. J., MacKintosh, F. C., Lubensky, T. C. \& Janmey, P. A Nonlinear elasticity in biological gels. Nature 435, 191-194 (2005).

43. Uzel, S. G. M. et al. Simultaneous or sequential orthogonal gradient formation in a $3 \mathrm{D}$ cell culture microfluidic platform. Small 12, 612-622 (2016).

44. Manfrin, A. et al. Engineered signaling centers for the spatially controlled patterning of human pluripotent stem cells. Nat. Methods 16, 640-648 (2019).

45. Cederquist, G. Y. et al. Specification of positional identity in forebrain organoids. Nat. Biotechnol. 37, 436-444 (2019).

46. DeForest, C. A. \& Anseth, K. S. Photoreversible patterning of biomolecules within click-based hydrogels. Angew. Chemie Int. Ed. 51, 1816-1819 (2012).

47. Guvendiren, M. \& Burdick, J. A. Stiffening hydrogels to probe short- and long-term cellular responses to dynamic mechanics. Nat. Commun. 3, 792 (2012).

48. Vincent, L. G., Choi, Y. S., Alonso-Latorre, B., Del Álamo, J. C. \& Engler, A J. Mesenchymal stem cell durotaxis depends on substrate stiffness gradient strength. Biotechnol. J. 8, 472-484 (2013).

49. Xia, B. \& Yanai, I. A periodic table of cell types. Development 146, dev169854 (2019).

50. Roost, M. S. et al. KeyGenes, a tool to probe tissue differentiation using a human fetal transcriptional atlas. Stem Cell Reports 4, 1112-1124 (2015)

51. Nowotschin, S. et al. The emergent landscape of the mouse gut endoderm at single-cell resolution. Nature 569, 361-367 (2019).

52. Pijuan-Sala, B. et al. A single-cell molecular map of mouse gastrulation and early organogenesis. Nature 566, 490-495 (2019).

53. Mayr, U., Serra, D. \& Liberali, P. Exploring single cells in space and time during tissue development, homeostasis and regeneration. Development 146 , dev176727 (2019).

54. Hannezo, E. \& Heisenberg, C. P. Mechanochemical feedback loops in development and disease. Cell 178, 12-25 (2019).

55. Bailles, A. et al. Genetic induction and mechanochemical propagation of a morphogenetic wave. Nature 572, 467-473 (2019).

56. Alt, S., Ganguly, P. \& Salbreux, G. Vertex models: from cell mechanics to tissue morphogenesis. Philos. Trans. R. Soc. B 372, 20150520 (2017)

57. Latorre, E. et al. Active superelasticity in three-dimensional epithelia of controlled shape. Nature 563, 203-208 (2018).

58. Okuda, S. et al. Strain-triggered mechanical feedback in self-organizing optic-cup morphogenesis. Sci. Adv. 4, eaau1354 (2018).

59. Karzbrun, E., Kshirsagar, A., Cohen, S. R., Hanna, J. H. \& Reiner, O. Human brain organoids on a chip reveal the physics of folding. Nat. Phys. 14, 515-522 (2018)

60. Bershteyn, M. et al. Human iPSC-derived cerebral organoids model cellular features of lissencephaly and reveal prolonged mitosis of outer radial glia. Cell Stem Cell 20, 435-449 (2017).

61. Forbes, T. A. et al. Patient-iPSC-derived kidney organoids show functional validation of a ciliopathic renal phenotype and reveal underlying pathogenetic mechanisms. Am. J. Hum. Genet. 102, 816-831 (2018).

62. Freedman, B. S. et al. Modelling kidney disease with CRISPR-mutant kidney organoids derived from human pluripotent epiblast spheroids. Nat. Commun. 6, 8715 (2015).

63. Bian, S. et al. Genetically engineered cerebral organoids model brain tumor formation. Nat. Methods 15, 631-639 (2018).

64. Ogawa, J., Pao, G. M., Shokhirev, M. N. \& Verma, I. M. Glioblastoma model using human cerebral organoids. Cell Rep. 23, 1220-1229 (2018).

65. Qian, X. et al. Brain-region-specific organoids using mini-bioreactors for modeling ZIKV exposure. Cell 165, 1238-1254 (2016).

66. Przepiorski, A. et al. A simple bioreactor-based method to generate kidney organoids from pluripotent stem cells. Stem Cell Rep. 11, 470-484 (2018).

67. Wimmer, R. A. et al. Human blood vessel organoids as a model of diabetic vasculopathy. Nature 565, 505-510 (2019).

68. Sabbagh, M. F. et al. Transcriptional and epigenomic landscapes of CNS and non-CNS vascular endothelial cells. eLife 7, e36187 (2018).

69. Takebe, T. et al. Vascularized and complex organ buds from diverse tissues via mesenchymal cell-driven condensation. Cell Stem Cell 16, 556-565 (2015).
70. Czerniecki, S. M. et al. High-throughput screening enhances kidney organoid differentiation from human pluripotent stem cells and enables automated multidimensional phenotyping. Cell Stem Cell 22, 929-940 (2018).

71. Taguchi, A. \& Nishinakamura, R. Higher-order kidney organogenesis from pluripotent stem cells. Cell Stem Cell 21, 730-746 (2017).

72. Mansour, A. A. et al. An in vivo model of functional and vascularized human brain organoids. Nat. Biotechnol. 36, 432-441 (2018).

73. Takebe, T. et al. Vascularized and functional human liver from an iPSC-derived organ bud transplant. Nature 499, 481-484 (2013).

74. Takebe, T. et al. Massive and reproducible production of liver buds entirely from human pluripotent stem cells. Cell Rep. 21, 2661-2670 (2017)

75. Van den Berg, C. W. et al. Renal subcapsular transplantation of PSC-derived kidney organoids induces neo-vasculogenesis and significant glomerular and tubular maturation in vivo. Stem Cell Rep. 10, 751-765 (2018).

76. Campisi, M. et al. 3D self-organized microvascular model of the human blood-brain barrier with endothelial cells, pericytes and astrocytes. Biomaterials 180, 117-129 (2018).

77. Shirure, V. S. et al. Tumor-on-a-chip platform to investigate progression and drug sensitivity in cell lines and patient-derived organoids. Lab Chip 18, 3687-3702 (2018)

78. Song, J., Miermont, A., Lim, C. T. \& Kamm, R. D. A 3D microvascular network model to study the impact of hypoxia on the extravasation potential of breast cell lines. Sci. Rep. 8, 17949 (2018)

79. Homan, K. A. et al. Flow-enhanced vascularization and maturation of kidney organoids in vitro. Nat. Methods 16, 255-262 (2019).

80. Mark, A. et al. Biomanufacturing of organ-specific tissues with high cellular density and embedded vascular channels. Sci. Adv. 5, eaaw2459 (2019).

81. Noor, N. et al. 3D personalized thick and perfusable cardiac patches and hearts. Adv. Sci. 6, 1900344 (2019).

82. Workman, M. J. et al. Engineered human pluripotent-stem-cell-derived intestinal tissues with a functional enteric nervous system. Nat. Med. 23, 49-59 (2017).

83. Birey, F. et al. Assembly of functionally integrated human forebrain spheroids. Nature 545, 54-59 (2017).

84. Koike, H. et al. Modelling human hepato-biliary-pancreatic organogenesis from the foregut-midgut boundary. Nature 574, 112-116 (2019).

85. Esch, M. B., Mahler, G. J., Stokol, T. \& Shuler, M. L. Body-on-a-chip simulation with gastrointestinal tract and liver tissues suggests that ingested nanoparticles have the potential to cause liver injury. Lab Chip 14, 3081-3092 (2014)

86. Bauer, S. et al. Functional coupling of human pancreatic islets and liver spheroids on-a-chip: Towards a novel human ex vivo type 2 diabetes model. Sci. Rep. 7, 14620 (2017).

87. Osaki, T., Uzel, S. G. M. \& Kamm, R. D. Microphysiological 3D model of amyotrophic lateral sclerosis (ALS) from human iPS-derived muscle cells and optogenetic motor neurons. Sci. Adv. 4, eaat5847 (2018).

88. Achberger, $\mathrm{K}$. et al. Merging organoid and organ-on-a-chip technology to generate complex multi-layer tissue models in a human retina-on-a-chip platform. eLife 8, e46188 (2019).

89. Tao, T. et al. Engineering human islet organoids from iPSCs using an organ-on-chip platform. Lab Chip 19, 948-958 (2019).

90. Workman, M. J. et al. Enhanced utilization of induced pluripotent stem cell-derived human intestinal organoids using microengineered chips. Cell. Mol. Gastroenterol. Hepatol. 5, 669-677 (2018).

91. Lee, K. K. et al. Human stomach-on-a-chip with luminal flow and peristaltic-like motility. Lab Chip 18, 3079-3085 (2018).

92. Wang, Y. et al. In situ differentiation and generation of functional liver organoids from human iPSCs in a 3D perfusable chip system. Lab Chip $\mathbf{1 8}$ 3606-3616 (2018)

93. Bredenoord, A. L., Clevers, H. \& Knoblich, J. A. Human tissues in a dish: the research and ethical implications of organoid technology. Science 355, eaaf9414 (2017).

94. Munsie, M., Hyun, I. \& Sugarman, J. Ethical issues in human organoid and gastruloid research. Development 144, 942-945 (2017).

95. Hyun, I. Engineering ethics and self-organizing models of human development: opportunities and challenges. Cell Stem Cell 21, 718-720 (2017).

96. Van de Poel, I. \& van Gorp, A. C. The need for ethical reflection in engineering design. Sci. Technol. Hum. Values 31, 333-360 (2006).

97. Sample, M. et al. Multi-cellular engineered living systems: building a community around responsible research on emergence. Biofabrication 11, 043001 (2019).

98. Chuva de Sousa Lopes, S. M. Accelerating maturation of kidney organoids. Nat. Mater. 18, 303-304 (2019).

99. Miura, Y. \& Pașca, S. P. Polarizing brain organoids. Nat. Biotechnol. 37, 377-378 (2019)

100. Wilson, H. V. On some phenomena of coalescence and regeneration in sponges. J. Exp. Zool. 5, 245-258 (1907). 
101. Harrison, R. G. Observations on the living developing nerve fiber. Exp. Biol. Med. 4, 140-143 (1906).

102. Strangeways, T. S. P. \& Fell, H. B. Experimental studies on the differentiation of embryonic tissues growing in vivo and in vitro.-II. The development of the isolated early embryonic eye of the fowl when cultivated in vitro. Proc. R. Soc. B Biol. Sci. 100, 273-283 (1926).

103. Trowell, O. A. A modified technique for organ culture in vitro. Exp. Cell Res. 6, 246-248 (1954).

104. Streuli, C. H. \& Bissell, M. J. Expression of extracellular matrix components is regulated by substratum. J. Cell Biol. 110, 1405-1415 (1990).

105. Roca-Cusachs, P., Conte, V. \& Trepat, X. Quantifying forces in cell biology. Nat. Cell Biol. 19, 742-751 (2017).

106. Grashoff, C. et al. Measuring mechanical tension across vinculin reveals regulation of focal adhesion dynamics. Nature 466, 263-266 (2010).

107. Legant, W. R. et al. Measurement of mechanical tractions exerted by cells in three-dimensional matrices. Nat. Methods 7, 969-971 (2010).

108. Campàs, O. et al. Quantifying cell-generated mechanical forces within living embryonic tissues. Nat. Methods 11, 183-189 (2014).

109. Kumar, S. et al. Viscoelastic retraction of single living stress fibers and its impact on cell shape, cytoskeletal organization, and extracellular matrix mechanics. Biophys. J. 90, 3762-3773 (2006).

110. Etournay, R. et al. TissueMiner: a multiscale analysis toolkit to quantify how cellular processes create tissue dynamics. eLife 5, e14334 (2016).

\section{Acknowledgements}

We thank SOLIDCAM ESTUDIO for support with figure illustrations. This work has received funding from the European Research Council (ERC) under the European Union's Horizon 2020 research and innovation program (StG-2014-640525 REGMAMKID to N.M. and FOR EXAMPLE, CoG-2013-616480_TensionControl to X.T., CoG-2016-725722_OVOGROWTH to S.M.C.S.L. and StG-757710 to M.A.L.). E.G. is funded by the EFSD/Boehringer Ingelheim European Research Programme in Microvascular Complications of Diabetes. R.D.K. received support from the US National Science Foundation (CBET-0939511). M.A.L. received funding from the Medical Research Council (MC_UP_1201/9). R.W. received support from the NIH (R01EB024591 and R01EB025256) and the NSF (0939511 and 1446474) research grants. X.T. is also supported by the European Commission (project H2O 20-FETPROACT-01-2016-731957). I.H. is funded by the Greenwall Foundation's 'Making a Difference' grant. This work also received funding from the Spanish Ministry of Economy and Competitiveness (MINECO)/FEDER (SAF2015-72617-EXP to N.M. and SAF2017-89782-R to N.M. and PGC2018-099645-B-I00 to X.T.), Generalitat de Catalunya and CERCA programme (2017 SGR 1306 to N.M. and SGR-2017-01602 to X.T.), Asociación Española contra el Cáncer (AECC) (LABAE16006 to N.M.). N.M. is also supported CardioCel (TerCel, Instituto de Salud Carlos III). The Institute for Bioengineering of Catalonia is the recipient of a 'Centro de Excelencia Severo Ochoa' award from the MINECO (funded by the Agencia Estatal de Investigación: SEV20140425 and CEX2018-000789-S) and MIT-Spain 'la Caixa' Foundation Seed Fund award 'Bioenginering Against Cancer'.

\section{Author contributions}

N.M. conceived the outline of the Review, and wrote and revised the manuscript. E.G. wrote and revised the manuscript. R.D.K. contributed in the section on engineering vascularization and commented on the manuscript. S.M.C.S.L. contributed on the section of single-cell transcriptomics and commented on the manuscript. M.A.L. contributed to the section of understanding self-organization and symmetry breaking. R.W. commented on the manuscript. X.T. contributed to the section of probing mechanics in hPSC-organoids. I.H. wrote the section of engineering ethics and commented on the manuscript. N.M. and E.G. created the figures.

\section{Competing interests}

R.D.K. is co-founder and has a substantial financial interest in AIM Biotech, and receives research support from Amgen, Biogen and Gore.

\section{Additional information}

Correspondence should be addressed to N.M

Reprints and permissions information is available at www.nature.com/reprints.

Publisher's note Springer Nature remains neutral with regard to jurisdictional claims in published maps and institutional affiliations.

๑) Springer Nature Limited 2020 


\section{QUERY FORM}

\begin{tabular}{|l|c|}
\hline \multicolumn{2}{|c|}{ Nature Materials } \\
\hline Manuscript ID & [Art. Id: 804] \\
\hline Author & Elena Garreta \\
\hline
\end{tabular}

\section{AUTHOR:}

The following queries have arisen during the editing of your manuscript. Please answer by making the requisite corrections directly in the e.proofing tool rather than marking them up on the PDF. This will ensure that your corrections are incorporated accurately and that your paper is published as quickly as possible.

\begin{tabular}{|c|c|}
\hline Query No. & Nature of Query \\
\hline Q1: & What does 'Pluripotency for Organ Regeneration' represent in affiliation 1? Is it the name of a research group? \\
\hline Q2: & $\begin{array}{l}\text { Has the separation of list items been interpreted correctly in the sentence beginning 'These include the lack'? } \\
\text { Semicolons have been used because one item in the list already contains a comma ('absence of proper vascular, } \\
\text { immune and innervation components and organ-specific morphological features'). }\end{array}$ \\
\hline Q3: & $\begin{array}{l}\text { Please check your article carefully, coordinate with any co-authors and enter all final edits clearly in the eproof, } \\
\text { remembering to save frequently. Once corrections are submitted, we cannot routinely make further changes to } \\
\text { the article. }\end{array}$ \\
\hline Q4: & $\begin{array}{l}\text { Note that the eproof should be amended in only one browser window at any one time; otherwise changes will } \\
\text { be overwritten. }\end{array}$ \\
\hline Q5: & $\begin{array}{l}\text { Author surnames have been highlighted. Please check these carefully and adjust if the first name or surname } \\
\text { is marked up incorrectly. Note that changes here will affect indexing of your article in public repositories such } \\
\text { as PubMed. Also, carefully check the spelling and numbering of all author names and affiliations, and the cor- } \\
\text { responding email address(es). }\end{array}$ \\
\hline Q6: & $\begin{array}{l}\text { There should be some text between level } 1 \text { headings and level } 2 \text { headings. Please add some text where indicated } \\
\text { after the 'Engineering human pluripotent stem cell derived organoids' heading. }\end{array}$ \\
\hline Q7: & $\begin{array}{l}\text { We reserve the word significant and its derivatives to mean statistically significant, in which case we would } \\
\text { require P values. Instances of significant and significantly have been changed to substantial and substantially } \\
\text { throughout, as it didn't seem appropriate to include P values. }\end{array}$ \\
\hline Q8: & Note that we no longer make common latin phrases like in vitro italic. We also don’t use italics for emphasis. \\
\hline Q9: & $\begin{array}{l}\text { Note that we use single quote marks for figures of speech and titles, and double quote marks for direct quota- } \\
\text { tions of speech. Please check this is consistent throughout, particularly in Box } 1 .\end{array}$ \\
\hline Q10: & $\begin{array}{l}\text { References that appear only in boxes are numbered after those that appear only in figure captions. Refs } 98- \\
110 \text { have been renumbered slightly to reflect this; please check that each citation corresponds to the correct } \\
\text { reference. }\end{array}$ \\
\hline Q11: & Has FRET been expanded correctly as 'Förster resonance energy transfer'? \\
\hline Q12: & In the acknowledgements, M.L. was changed to M.A.L. to match the author list-OK? \\
\hline
\end{tabular}

